\title{
Pedantic Speaking Style Differentiates Asperger Syndrome from High-Functioning Autism ${ }^{1}$
}

\author{
Mohammad Ghaziuddin ${ }^{2}$ and Leonore Gerstein \\ University of Michigan, Ann Arbor
}

Asperger syndrome $(A S)$ is a pervasive developmental disorder recently introduced as a new diagnostic category in the ICD-10 and the DSM-IV. Along with motor clumsiness, pedantic speech has been proposed as a clinical feature of $A S$. However, few attempts have been made to define and measure this symptom. We studied 17 patients with $A S$ (ICD-10; 14 male, 3 female; mean age 16.4 years, mean full-scale IQ 97) and compared them with a control group of 13 patients with normal-intelligence autism or high-functioning autism (HFA) (ICD-10/DSM-III-R; 12 male, 1 female; mean age 15.5 years, mean full-scale IQ 81.2). An operational definition of pedantic speech was formulated and a rating scale devised. $13(76 \%)$ of the $A S$ patients were rated as pedantic compared to $4(31 \%)$ of the HFA group $\left(\chi^{2}=6.3 ; \mathrm{p}=.01\right)$. Results suggest that pedantic speech is common in $A S$ and may help differentiate $A S$ from high-functioning autism.

\section{INTRODUCTION}

Asperger syndrome (AS) is a pervasive developmental disorder characterized by social deficits, relatively normal language and cognitive development,

\footnotetext{
${ }^{1}$ The authors acknowledge the contribution of Michael Clark, Professor of Speech Pathology, Western Michigan University, Kalamazoo, Michigan; Cynthia McLemore, Stanford University, California; Tami Benedek, speech pathologist, University of Michigan; and John F. Greden. Elizabeth Hill assisted with the data analysis. Our thanks also to Sir Michael Rutter, who commented on an earlier draft. This paper was completed, in part, while the first author was on an academic visit to the Institute of Psychiatry, London, sponsored by a University of Michigan OVPAMA faculty award.

${ }^{2}$ Address all correspondence to Mohammad Ghaziuddin, Taubman Center, Box 0390, University of Michigan Medical Center, 1500 East Medical Center Drive, Ann Arbor, Michigan 48109-0390.
} 
and the presence of idiosyncratic interests. Although first described by Asperger in 1944, AS has only recently been included in the ICD-10 (World Health Organization [WHO], 1993) and in the newly released DSM-IV (American Psychiatric Association [APA], 1994) as a distinct category within the pervasive developmental disorders.

Despite occasional past interest in AS (e.g., Van Krevelen, 1971), not much attention was focused upon it until Wing (1981) described its clinical features in a series of 34 patients ranging in age from 5 to 35 years. Since then, there has been a marked resurgence of interest in this disorder. However, despite its inclusion as a separate diagnostic category, its distinction from autism with normal intelligence, so-called high-functioning autism (HFA), continues to be a matter of debate. Asperger syndrome has been proposed as part of the "autistic continuum" with AS at the upper end, autism in the middle, and severely retarded children with the triad of language and social impairment at the lower end (Gillberg, 1989). Although some authorities have cautioned against its premature acceptance as a valid entity (Schopler, 1985), others have suggested that AS may be clinically differentiated from autism. For example, Ozonoff, Rogers, and Pennington (1991) found that patients with AS performed better than HFA controls on theory of mind and verbal memory tests. Also, children with AS have been described as being more clumsy than children with autism, although evidence for the diagnosis of AS based on this psychomotor distinction is not well established at this stage (Ghaziuddin, Butler, Tsai, \& Ghaziuddin, 1994; Ghaziuddin, Tsai, \& Ghaziuddin, 1992a). Establishing the diagnostic validity of AS may also be important to alert clinicians to potential comorbidity issues. For example it has been suggested that AS may be associated with increased psychiatric morbidity (Wing, 1981), especially mood disorders (Gillberg, 1985; DeLong \& Dwyer, 1988). Also, AS may have an overlap with schizoid/schizotypal personality disorder (Wolff \& Barlow, 1979) and with nonverbal learning disability (Stevens \& Moffit, 1988). Thus, further phenomenological investigations of AS are clearly indicated (see Rutter \& Schopler, 1992).

Possible reasons for the confusion surrounding the nosological status of Asperger syndrome are the lack of consensus about its clinical features and the use of a variety of diagnostic criteria (see Ghaziuddin, Tsai, \& Ghaziuddin, 1992b). Along with clumsiness, several investigators have remarked on the presence of pedantic speech in persons with AS (Wing, 1981). Asperger himself used the term "pedantic" to refer to narrow and intense interests rather than speech (Frith, 1991). His characterization of speech ãs containing "unusual words. . newly formed or partially restructured expressions. . .often quite abstruse" (p. 71) appears to coincide with what later workers have called pedantic. Wing (1981) referred to pedantic 
lengthy speech, having a "bookish" quality, as a characteristic of the syndrome. She pointed out that persons with AS often use obscure words while simpler vocabulary may be deficient, and may appear to be inappropriately copying the speech of others. In a later paper, she suggested that pedantic speech formed one of the major clinical features of this syndrome (Burgoine \& Wing, 1983).

Others have described the style of individuals with semantic-pragmatic deficits in terms that are consistent with a pedantic quality, without labeling it as such. Tantam $(1986,1988)$ found a high prevalence $(67 \%)$ of pragmatic abnormalities in his group of patients with Asperger syndrome compared to a control group of patients with schizoid, schizotypal, and borderline personality disorders (14\%). In their examination of utterances of children with a semantic-pragmatic disorder, Bishop and colleagues (Adams \& Bishop, 1989; Bishop, 1989; Bishop and Adams, 1989) identified several types of "inappropriacy" including "too much information" and "socially inappropriate style." However, they did not refer to these features as being pedantic. Fine, Bartolucci, Szatmari, and Ginsberg (1994) noted that subjects with AS had difficulty monitoring their listeners' informational needs. Although the authors did not link this deficit with a pedantic style, we suspect that it contributes to poor conversational skills in general and to pedantic speech in particular. Eales (1993) used the semantic-pragmatic categories developed by Bishop and Adams (1989) and Adams and Bishop (1989) and found adults with autism experienced difficulty in forming context-relevant communicative intentions which resulted in their pragmatic impairment. However, neither he nor Bishop or Adams called any of the behaviors they coded "pedantic." Baltaxe and D'Angiola (1992) proposed that insufficient or incorrect use of ellipses typifies speakers with autism. Similarly, in their investigation of the speaking style of parents of autistic individuals, Landa et al. (1992) included overly talkative, overly detailed topic preoccupation, insufficient background information, and little "to and fro" among the features of deficient communication. However, it is not clear if these speech abnormalities are similar to those seen in persons with AS and, also, if a specific link exists between pedantic speech and this syndrome. A review of the literature suggests that, despite the reported association between pedantic speech and AS, to our knowledge, no systematic attempt has been made to define and measure this symptom. If indeed pedantic speech is common in AS, it would be useful to investigate its utility as a diagnostic feature of this disorder. The present study was, therefore, undertaken with the following aims (a) to operationally define and measure the presence of pedantic speech in patients with AS and matched controls; and (b) to investigate if pedantic speech can differentiate between Asperger syndrome and high-functioning autism. 
Table I. ICD-10 Criteria (1993) for Asperger Syndrome

A. A lack of any clinically significant general delay in language or cognitive development. Diagnosis requires that single words should have developed by 2 years of age or earlier and that communicative phrases be used by 3 years of age or earlier. Motor clumsiness is usual, although not a necessary diagnostic feature. Isolated special skills, often related to abnormal preoccupations, are common, but are not required for diagnosis.

B. Qualitative impairments in reciprocal social interaction (as in autism).

C. Restricted, repetitive, and stereotyped patterns of behavior, interests, and activities (criteria as for autism; however, it would be less usual for these to include either motor mannerisms or preoccupations with part-objects or nonfunctional elements of play materials).

D. The disorder is not attributable to the other varieties of pervasive developmental disorder; schizotypal disorder; simple schizophrenia; reactive and disinhibited attachment disorder of childhood; obsessional personality disorder; and obsessive-compulsive disorder.

\section{METHOD}

The study was conducted at the University of Michigan Developmental Disorders Clinic. The sample consisted of patients referred consecutively to the clinic. Diagnosis of pervasive developmental disorders was made after a comprehensive multidisciplinary evaluation which consisted of semistructured interviews with parents and patients, speech and language evaluation, psychological testing, and behavioral assessment. The clinical information was supplemented by data based on the Autism Behavior Checklist (Krug, Arick, \& Almond, 1980), and the Vineland Adaptive Behavior Scales (Sparrow, Balla, \& Cicchetti, 1984). All available written records from educational agencies, social workers, and schools were also reviewed.

Patients with AS consisted of those who met the criteria for that disorder as laid down by the ICD-10 (WHO, 1988). These were patients with pervasive developmental disorders who failed to meet the criteria for ICD10 autism (or of DSM-III-R [APA, 1987] autistic disorder) but suffered from autistic social dysfunction and idiosyncratic interests in the presence of a full-scale IQ of 70 or above on an individually administered test of intelligence (WISC-R; Wechsler, 1974) or (WAIS, Wechsler, 1981). None of them had a history of speech delay ( $n=17 ; 14$ males; $M$ age: 16.4 years; $M$ full-scale IQ: 97$) \cdot{ }^{3}$ It is important to note that none of the AS subjects had met the criteria for autism in the past (see Table I).

\footnotetext{
${ }^{3}$ The study was completed before the final version of the ICD-10 criteria was published (1993); however, the diagnostic criteria of AS given in the ICD-10 draft version (1988) and those given in the final version (1993) are virtually identical.
} 
The control group of patients with HFA were referred to the same clinic over the same index period. They met the DSM-III-R/ICD-10 criteria for autism and had a full-scale IQ over $70(n=13 ; 12$ males; $M$ age: 15.5 years; $M$ full-scale IQ: 81.2). The two groups resembled each other in age and sex but differed significantly in their full-scale IQ $(t=2.8 ; p=.01)$ and in their verbal IQ $(t=2.7 ; p=.01)$. Verbal/performance IQ scores were not available in one control.

English was the native language of both the groups of patients. One patient with AS was African American; all other subjects were Caucasian and were born in the United States. None suffered from any current seizure disorder or other diagnosed neurologic disorder (such as tuberous sclerosis) known to coexist with autism. None of the subjects or controls met the clinical description of fragile $\mathrm{X}$ syndrome.

The definition of pedantic speech was based on the meaning of the word pedant (Oxford English Dictionary, 1971). A pedant is described as: "1. a school master or teacher; 2. a person who overrates book-learning or technical knowledge or displays it unduly or unreasonably; one who has mere learning untempered by practical judgment and knowledge of affairs; or who lays excessive stress upon trifling details of knowledge or upon strict adherence to formal rules; sometimes one who is possessed by a theory and insists on applying it in all cases without discrimination." Thus, pedantic speech may be defined as that type of speech in which the speaker conveys more information than the topic and goals of the conversation demand, violating expectations of relevancy and quantity; sentence structure may have the formality, and vocabulary display the erudition expected of written language. Conversational turns resemble rehearsed monologues rather than contributions to a jointly managed dialogue. Articulation may be precise and intonation formal. The description of conversational inappropriacy as proposed by Bishop and Adams (1989) also contributed to our definition of pedantic speech. Particularly relevant was their formulation of the categories of "too much information" and "unusual or socially inappropriate content or style," the former referring to unnecessary elaboration or reiteration and the latter to stereotyped or formulaic language. However, our definition and measures of pedantic speech do not mirror previously reported analyses of semantic-pragmatic impairments. Since our objective was to attempt to pinpoint not only the pragmatic but also the semantic, syntactic, and phonetic aspects associated with pedantry, we tried to avoid descriptions of general conversational features, such as "poor topic shifting," in favor of more narrowly defined behaviors. ${ }^{4}$

${ }^{4}$ Appendix can be obtained from author upon request. 
To systematically measure pedantic speaking style, one of us (L.G.), a certified speech and language pathologist, devised a rating scale that captured the relevant aspects of the dictionary definition, but more explicitly described the semantic, syntactic, pragmatic and, to a lesser degree, phonetic features of pedantic speech (see footnote 4). The scale was derived largely from clinical observations and from concepts in the field of conversational analysis (Ochs, 1979; Prutting \& Kirchner, 1987). Grice's (1975) maxims of conversation form the basis of several of its items, particularly the maxims of quantity and manner. ${ }^{5}$

The second author (L.G.) also determined whether or not the subjects were pedantic. She was blind to the subjects' diagnoses, and had met only two of them. Judgment of speaking style was based upon audition of 7- to 20-minute tape-recorded speech samples collected by the first author during structured taped interviews. Interviews included a picture-description task, followed by a semistructured session consisting of open-ended questions focusing on the subject's daily activities, hobbies, and other interests. The same picture-description task was performed by all subjects except for one patient with AS who participated only in the semistructured session. Judgment was based on acoustic data only, with no attempt to conduct an analysis of the speech samples in transcribed form. Subjects were rated as not pedantic, mildly pedantic, or pedantic (see Footnote 4). Because of the small numbers involved in each category, the latter two categories were collapsed for analysis.

\section{Data Analysis}

Differences between groups were tested using $t$ tests for continuous measures (i.e., IQ scores) or chi-square tests for categorical measures (i.e., presence or absence of pedantic speech). Because the diagnostic groups differed in IQ scores, we also tested for a confounding relationship between IQ and pedantic speech using a Pearson correlation. Tests were conducted using the Statview II statistical computer program (Abacus Concepts, Berkeley, CA).

\footnotetext{
${ }^{5}$ Maxim of quantity states that one should make one's contributions only as informative as is required; and according to the maxim of manner, one should be brief and orderly and avoid obscurity (Grice, 1975; Levinson, 1983).
} 


\section{RESULTS}

The two diagnostic groups differed significantly in their total pedantic speech scores, with the AS group scoring higher than the HFA group $(M=3.9$ AS; $1.4 \mathrm{HFA}), t(28)=2.8, p=.009$. Using a cutoff of 2 points as a definition of being categorized as pedantic, 13 of $17(76 \%)$ patients with AS were judged as such, compared to 4 of $13(31 \%)$ in the HFA group $\left(\chi^{2}=6.266, p=.0123\right)$. Verbal IQ scores were higher in the AS than the HFA groups ( $M=99.4 \mathrm{AS} ; 81.2 \mathrm{HFA}), t(27)=2.7, p=.011$. However, verbal intelligence scores were not higher for pedantic patients $(M=95$, pedantic; 87.5 , not pedantic), $t(27)=1.0, p=.322)$. The Pearson correlation between verbal IQ and pedantic scale score was also nonsignificant $(r=.2, p=.322)$. This relationship was found within the AS group as well; that is, patients with AS with high verbal IQ scores were not more likely to be rated as pedantic or score high on the pedantic scale, compared to those who did not have a high verbal IQ score. To clarify this issue further, an analysis of covariance was done using SAS package (SAS Institute, 1993). Homogeneity of slopes for the two groups was checked and after adjusting for verbal IQ, significant differences were found between the means of the total pedantic speech scores of the two groups, $F(1,26)=5.40, p=.0283$. Similarly, when scores on the pedantic scale were correlated with age of the whole sample, no correlation was found $(r=.3 ; p=.19)$. The mean number of utterances of the two groups were also compared. The AS group had a somewhat higher mean number of utterances than the HFA group (74.4 vs. 58.1), $t(28)=2, p=.06$. However, as a group, pedantic speakers produced more utterances than nonpedantic speakers irrespective of the diagnosis (74.3 vs. 58.2$), t(28)=1.9$, $p=.06$, suggesting that the HFA group was not penalized for "poverty of speech." Subsequently, to obtain a preliminary estimate of the reliability of the scale, 15 randomly selected speech samples from the 30 subjects were rated blindly by another certified speech pathologist. Agreement was found on 11 patients. Of the 7 patients rated as pedantic and 8 as not pedantic by L.G., 4 were rated as pedantic and 7 as not pedantic, respectively, by the other speech pathologist; no agreement was found on the remaining 4 patients $($ kappa $=.455$; agreement $=73 \%$ ).

\section{DISCUSSION}

This study makes a preliminary attempt to operationally define and quantify the presence of pedantic speech in AS. Of the AS sample, 76\% was categorized as being pedantic based on the definition and the rating 
scale used in this study, compared to $31 \%$ of the controls with HFA. Significant differences were also found between patients with AS and HFA in their total pedantic speech scores, with the AS group scoring higher than the HFA group. Before drawing any firm conclusions, however, the following limitations should be emphasized. First, the study was based on a modest-sized clinic sample; therefore, generalizations are not yet appropriate. Second, it should be noted that the scale used is preliminary in nature and that its reliability and validity are yet to be formally established. Third, since rating of the speech samples was based entirely on audio samples, it is conceivable that in some patients, a face-to-face interview may have provided extra opportunities for patients to show more pedantic features, thereby resulting in higher pedantic scores. Similarly, the duration of the tape-recorded interview may have had an impact on whether or not the patient was categorized as being pedantic, although the HFA group did not appear to be penalized for their somewhat lower mean number of utterances because the pedantic speakers, irrespective of the diagnosis, had a higher mean number of utterances than the nonpedantic speakers. Finally, although the overall scores of the AS group were higher than the HFA group, 4 autistic patients were also categorized as pedantic. Although no group differences characterized these patients, it is well known that some autistic persons may also speak in a "stilted" manner (Rutter, 1965). Thus, larger samples are needed to clarify this issue further.

Four patients with AS were not rated as pedantic. This serves to emphasize the fact that, even though as a group AS patients may show a tendency to pedantic speech, the diagnosis cannot be based on the presence of a single criterion and should take all factors into consideration, such as the degree of social impairment, history of language acquisition, cognitive level, and the presence of idiosyncratic interests. At the same time, other reasons may explain why the 4 AS patients were not rated as pedantic. First, the tendency to be pedantic may be more apparent in certain social situations. A familiar examiner and a relaxed unstructured encounter may be more appropriate, and even necessary, to allow some persons to talk about their favorite topics. A semistructured picture-and-story task, as the one used in the present study, may not be appropriate in all cases. Second, it is possible that the tendency to be pedantic increases both with age and with the fund of information collected over time. People with AS have a tendency to "specialize" in idiosyncratic interests. With time, some of them gather enormous amounts of factual information that they tend to use in social situations, with no regard to the interest of the listener, giving the impression of being pedantic. For this to occur, therefore, it is important that the person gets adequate exposure and access to his topic of interest. Since the diagnostic criteria for AS do not make an allowance for age, it 
is possible that circumscribed interests and pedantic speech do not develop till the child is at least in his adolescence (Ghaziuddin et al., 1992b). In this context, it is important to note that 2 of the 4 patients with AS rated as not pedantic were under 10 years of age. Patients with AS may, therefore, show a trend to be pedantic as they grow older, although in the present sample, we did not find any association between age and being categorized as pedantic or between age and continuous pedantic scores. Also, the tendency to appear pedantic did not seem to be dependent on the subjects' topic of interest, at least in this sample. Similarly, clinical experience suggests that the tendency to speak in a pedantic and formal manner may partly be due to the high verbal intelligence scores of the AS sample. However, when the results were analyzed, this was not found to be the case. The tendency to speak in a pedantic manner did not apparently reflect the high verbal intelligence scores of the AS sample, since as a group, patients with high verbal intelligence scores (both groups) were not more likely to be rated as pedantic. Again, because of the modest size of the sample, the role of higher verbal intelligence on the speaking style in AS needs to be explored in future studies.

If replicated, results of this pilot study raise the possibility that pedantic speaking style may be used as a diagnostic feature of AS. Distinction between autism and AS continues to be an important area of research. Asperger syndrome has aptly been described as a key to the puzzle of autism (Rutter, personal communication). However, although most authorities agree about its clinical usefulness, its diagnostic validity and its distinction from HFA are still not established. Since an important difference between AS and HFA at this stage seems to be the relatively normal language development in the former, it is clear that this distinction is largely dependent on parental recall. Use of a criterion, such as pedantic speech, that is not dependent on parental recall, may provide a useful method of establishing the diagnostic validity of Asperger syndrome and its possible distinction from high-functioning autism.

\section{REFERENCES}

Adams, C., \& Bishop, D. V. M. (1989). Semantic-pragmatic disorder: I. Exchange structure, turntaking, repairs and cohesion. British Joumal of Disorders of Communication, 24, 211-239.

American Psychiatric Association. (1987). Diagnostic and statistical manual of mental disorders (3rd ed. rev.). Washington, DC: Author.

American Psychiatric Association. (1994). Diagnostic and statistical manual of mental disorders (4th ed.). Washington, DC: Author.

Asperger, H. (1944). Die autischen psychopathen im kindesalter. Archiv fur Psychiatrie und Nervenkrankheiter, 117, 76-137. 
Baltaxe, C. A. M., \& d'Angiola, N. (1992). Cohesion in the discourse interaction of autistic, specifically language-impaired, and normal children. Journal of Autism and Developmental Disorders, 22, 1-22.

Bishop, D. V. M. (1989). Autism, Asperger syndrome and semantic-pragmatic disorder: Where are the boundaries? British Journal of Disorders of Communication, 24, 107-121.

Bishop, D. V. M., \& Adams, C. (1989). Conversational characteristics of children with semantic-pragmatic disorder: II. What leads to a judgment of inappropriacy? British Journal of Disorders of Communication, 24, 241-263.

Burgoine, E., \& Wing, L. (1983). Identical triplets with Asperger's syndrome. British Journal of Psychiatry, 143, 261-265.

DeLong, R. G., \& Dwyer, J. T. (1988). Correlation of family history with specific autistic subgroups: Asperger's syndrome and bipolar affective disease. Journal of Autism and Developmental Disorders, 18, 593-600.

Eales, M. (1993). Pragmatic impairments in adults with childhood diagnosis of autism or developmental receptive language disorder. Journal of Autism and Developmental Disorders, 23, 593-617.

Fine, J., Bartolucci, G., Szatmari, P., \& Ginsberg, G. (1994). Cohesive discourse in pervasive developmental disorders. Journal of Autism and Developmental Disorders, 24, 315-329.

Frith, U. (1991). Autism and Asperger syndrome. Cambridge, U.K.: Cambridge University Press.

Ghaziuddin, M., Butler, L., Tsai, L., \& Ghaziuddin, N. (1994). Is clumsiness a marker for Asperger syndrome? Joumal of Intellectual Disability Research, 38, 519-527.

Ghaziuddin, M., Tsai, L., \& Ghaziuddin, N. (1992a). A reappraisal of clumsiness as a diagnostic feature of Asperger syndrome. Joumal of Autism and Developmental Disorders, 22, 651-656.

Ghaziuddin, M., Tsai, L., \& Ghaziuddin, N. (1992b). A comparison of the diagnostic criteria for Asperger syndrome. Journal of Autism and Developmental Disorders, 22, 643-649.

Gillberg, C. (1985). Asperger's syndrome and recurrent psychosis - A case study. Journal of Autism and Developmental Disorders, 15, 389-397.

Gillberg, C. (1989). Asperger syndrome in 23 Swedish children. Developmental Medicine and Child Neurology, 31, 520-531.

Grice, H. P. (1975). Logic and conversation. In P. Cole \& J. L. Morgan (Eds.), Syntax and semantics 3: Speech acts. New York: Academic Press.

Krug, D. A., Arick, J. R., \& Almond, P. J. (1980). Behaviour checklist for identifying severely handicapped individuals with high levels of autistic behavior. Journal of Child Psychology and Psychiatry, 21, 221-229.

Landa, R., Piven, J., Wzorek, M. M., Gayle, J. O., Chase, G. A., \& Folstein, S. E. (1992). Social language use in parents of autistic individuals. Psychological Medicine, 22, 245-254

Levinson, S. C. (1983). Pragmatics. Cambridge, U.K.: Cambridge University Press.

Ochs, E. (1979). In T. Givon (Ed.), Planned and unplanned discourse, in syntax and semantics, Vol. 12: Discourse and syntax (pp. 51-80). New York, Academic Press.

Oxford English Dictionary. (1971). Compact edition. Oxford: Oxford University Press.

Ozonoff, S., Rogers, S., \& Pennington, B. (1991). Asperger's syndrome: Evidence of an empirical distinction from high-functioning autism. Journal of Child Psychology and Psychiatry, 32, 1107-1122.

Prutting, C. A., \& Kirchner, D. M. (1987). A clinical appraisal of the pragmatic aspects of language. Journal of Speech and Hearing Disorders, 52, 105-119.

Rutter, M. (1965). The influence of organic and emotional factors on the origins, nature and outcome of childhood psychosis. Developmental Medicine and Child Neurology, 7, 518-528.

Rutter, M., \& Schopler, E. (1992). Classification of pervasive developmental disorders: some concepts and practical considerations. Journal of Autism and Developmental Disorders, 22, 459-481.

SAS Institute, Inc. (1993). SAS User's guide, Cary, NC: Author.

Schopler, E. (1985). Convergence of learning disabilities, higher-level autism, and Asperger syndrome. Journal of Autism and Developmental Disorders, 15, 359. 
Sparrow, S., Balla, D., \& Cicchetti, D. (1984). Vineland Adaptive Behavior Scales. Circle Pines, MN: American Guidance Service.

Stevens, D. E., \& Moffit, T. E. (1988). Neuropsychological profile of an Asperger's syndrome case with exceptional calculating ability. Clinical Neuropsychologist, 2, 228-238.

Tantam, D. (1986). Eccentricity and autism. Unpublished Ph.D. thesis, University of London.

Tantam, D. (1988). Lifelong eccentricity and social isolation. II. Asperger's syndrome or Schizoid Personality Disorder. British Joumal of Psychiatry, 153, 783-791.

Van Krevelen, D. (1971). Early infantile autism and autistic psychopathy. Journal of Autism and Childhood Schizophrenia, 1, 82-86.

Wechsler, D. (1981). Wechsler Intelligence Scale for Adults. New York: Psychological Corp.

Wechsler, D. (1974). Wechsler Intelligence Scale for Children-Revised. New York: Psychological Corp.

Wing, L. (1981). Asperger's syndrome: A clinical account. Psychological Medicine, 11, 115-129.

Wolff, S., \& Barlow, A. (1979). Schizoid Personality Disorder in childhood: A comparative study of schizoid, autistic and normal children. Journal of Child Psychology and Psychiatry, $20,29-46$.

World Health Organization. (1988). The ICD-10 classification of mental and behavioural disorders (draft). Geneva: Author.

World Health Organization. (1993). The ICD-10 classification of mental and behavioural disorders, Diagnostic criteria for research. Geneva: Author. 